\title{
Mutational analysis of the TSC1 and TSC2 genes in a diagnostic setting: genotype - phenotype correlations and comparison of diagnostic DNA techniques in Tuberous Sclerosis Complex
}

\author{
Ozgur Sancak ${ }^{1}$, Mark Nellist ${ }^{1}$, Miriam Goedbloed ${ }^{1}$, Peter Elfferich ${ }^{1}$, Cokkie Wouters ${ }^{1}$, \\ Anneke Maat-Kievit ${ }^{1}$, Bernard Zonnenberg ${ }^{3}$, Senno Verhoef ${ }^{2}$, Dicky Halley ${ }^{1}$ \\ and Ans van den Ouweland ${ }^{*, 1}$
}

\author{
${ }^{1}$ Department of Clinical Genetics, Erasmus Medical Center, Rotterdam, The Netherlands; ${ }^{2}$ Family Cancer Clinic, \\ Netherlands Cancer Institute, Amsterdam, The Netherlands; ${ }^{3}$ TSC Clinic, Department of Medical Oncology, \\ University Medical Center Utrecht, Utrecht, The Netherlands
}

Tuberous sclerosis complex (TSC) is an autosomal dominant disorder characterised by the development of hamartomas in multiple organs and tissues. TSC is caused by mutations in either the TSC1 or TSC2 gene. We searched for mutations in both genes in a cohort of 490 patients diagnosed with or suspected of having TSC using a combination of denaturing gradient gel electrophoresis, single-strand conformational polymorphism, direct sequencing, fluorescent in situ hybridisation and Southern blotting. We identified pathogenic mutations in 362 patients, a mutation detection rate of $74 \%$. Of these 362 patients, 276 had a definite clinical diagnosis of TSC and in these patients 235 mutations were identified, a mutation detection rate of $85 \%$. The ratio of TSC2:TSC1 mutations was 3.4:1. In our cohort, both TSC1 mutations and mutations in familial TSC2 cases were associated with phenotypes less severe than de novo TSC2 mutations. Interestingly, consistent with other studies, the phenotypes of the patients in which no mutation was identified were, overall, less severe than those of patients with either a known TSC1 or TSC2 mutation.

European Journal of Human Genetics (2005) 13, 731-741. doi:10.1038/sj.ejhg.5201402

Published online 30 March 2005

Keywords: TSC1 and TSC2; tuberous sclerosis complex; genotype-phenotype correlation

\section{Introduction}

Tuberous sclerosis complex (TSC, MIM \#191100), affecting between $1 / 6000$ and $1 / 10000$ individuals, is an autosomal dominant disorder characterised by seizures, mental retardation and the development of hamartomas in multiple organs and tissues. ${ }^{1}$ In approximately two-thirds of cases

*Correspondence: Dr A van den Ouweland, Department of Clinical Genetics, Erasmus Medical Center, Dr Molewaterplein 50, 3015 GE Rotterdam, The Netherlands. Tel: + 3110 4087197;

Fax: + 3110 4089489; E-mail: a.vandenouweland@erasmusmc.nl Received 27 October 2004; revised 4 February 2005; accepted 8 February 2005 neither parent has signs of TSC and the disease is caused by a de novo mutation, indicating a high rate of spontaneous mutation in the causative genes, TSC1 and TSC2.

The TSC1 gene consists of a 3.4-kb open reading frame encoded by 21 exons, whereas the $5.4 \mathrm{~kb}$ open reading frame of the TSC2 gene is encoded by 41 exons. Loss of heterozygosity across the TSC1 and TSC2 loci in TSCassociated lesions indicates that TSC1 and TSC2 are tumor suppressor genes. ${ }^{2}$ The TSC1 and TSC2 gene products, hamartin and tuberin, form a complex ${ }^{3}$ that activates the GTPase activity of rheb, preventing the rheb-GTP-dependent stimulation of cell growth through mTOR. ${ }^{4}$ 
A broad spectrum of mutations has been identified in both genes. ${ }^{5-10}$ Interestingly, large rearrangements, missense mutations and in-frame deletions are very rare in the TSC1 gene. The majority of TSC patients have a mutation in the TSC2 gene. In de novo cases, mutations in the TSC2 gene are found two to ten times more often than TSC1 mutations. ${ }^{9-12}$ In contrast, in multi-generation families segregating TSC, approximately half show linkage to TSC1 and half to TSC $2 .{ }^{13}$ It has been suggested that this may be because TSC patients with a TSC1 mutation are less likely to be severely affected and therefore more likely to have offspring. ${ }^{12}$ Although initially the broad phenotypic and genotypic spectra of TSC made it difficult to establish an association, ${ }^{7,11}$ studies of larger cohorts indicated that patients with a TSC1 mutation were less likely to be mentally retarded, ${ }^{9}$ while patients with a TSC2 mutation had a higher number and/or severity of clinical features. ${ }^{10}$

We have performed a comprehensive screen for mutations at the TSC loci and compared the phenotypes within a large unrelated TSC patient cohort including the previously described group of patients. ${ }^{7}$ The majority of TSC patients in our cohort have a mutation in the TSC2 gene, and, overall, TSC2 mutations are associated with a more severe TSC phenotype. Our analysis confirms and extends the findings of previous studies.

\section{Materials and methods Patients}

Samples from patients with a (putative) diagnosis of TSC were obtained via several specialists in the Netherlands and abroad. A standardised clinical evaluation form was sent to all the referring clinicians. In familial cases, only index patients were used for phenotypic comparisons.

\section{Mutation analysis}

DNA was extracted from peripheral blood cells using standard techniques. Mutation analysis was performed using a combination of single-strand conformational polymorphism (SSCP), denaturing gradient gel electrophoresis (DGGE), direct sequencing, Southern blotting and fluorescence in situ hybridisation (FISH).

SSCP, Southern and FISH analyses were performed as described previously. ${ }^{14,15}$ For DGGE, primers were designed using the Ingeny DGGE Primer Design program (primer sequences available upon request), and pooled PCR products were run on urea/formamide denaturing gradient polyacrylamide gels at $60^{\circ} \mathrm{C}$ for $17 \mathrm{~h}$ (Ingeny phorU DGGE system). SSCP was performed on $50 \%$ of the patients and DGGE on $75 \%$ of the cohort. One exon in TSC1 and five exons in TSC2 were not suitable for DGGE and were sequenced. If no mutation was identified, Southern blotting and FISH analysis were undertaken. Direct sequencing of M13-tagged PCR products was carried out on an ABI3100 capillary sequencer using Big Dye Terminator v 3 chemistry (Applied Biosystems). A nucleotide change was classified as a polymorphism when this change was present in the patient together with a pathogenic mutation, when it was present in the DNA of the unaffected parent of the patient or when it had been classified as a polymorphism in the literature. A complete overview of our mutation analysis strategy is available upon request.

Total RNA was isolated from cultured patient fibroblasts. RT-PCR was performed according to standard procedures. Functional analysis of the effect of TSC 2 missense changes on the tuberin-hamartin complex was performed as described previously. ${ }^{16,17}$

\section{Statistical analysis}

Statistical comparisons were performed using the SPSS version 11.0 package for PC. Pearson $\chi^{2}$ or Fisher's exact test was used for comparisons of categorical data and Mann-Whitney test was used for the comparison of median age at referral.

\section{Results \\ Patient characteristics}

Mutation analysis of the TSC1 and TSC2 genes was performed on 490 patients with a (putative) diagnosis of TSC (Table 1). In 276 cases, sufficient clinical information was obtained to make a definite diagnosis of TSC according to the criteria defined by Roach et al. ${ }^{18}$ In 15 cases there was insufficient data for a definite diagnosis and in 199 cases we did not receive any clinical data. The 291 patients with clinical information were between 0 and 60 years old at the time of referral, with a median age of 13.0.

We examined the correlation between different clinical features in the 276 definitive TSC patients. The majority of the patients were mentally retarded (166/276; 60\%), and 163 of these patients ( $98 \%$ ) had seizures. In contrast, only 37 of the 63 patients without mental retardation had seizures $(59 \%)$. This difference was significant $(P<0.001)$. Seizures were present in $86 \%$ of the patients with (sub)cortical tubers and in $56 \%$ of the patients free of tubers $(P=0.004)$. The presence of (sub)cortical tubers also correlated with the presence of mental retardation. Of the patients with (sub)cortical tubers $82 \%$ had mental retardation, while only $33 \%$ of the patients without tubers were mentally retarded $(P=0.002)$. Subependymal nodules were noted in $88 \%$ of the patients with (sub)cortical tubers and in $63 \%$ of the patients without tubers $(P=0.013)$. In addition, there was a significant correlation between the incidence of renal angiomyolipomas and mental retardation in patients above the age of 6 years at referral. The patients with renal angiomyolipoma (82\%) had more frequently mental retardation compared to the patients without renal angiomyolipoma $(64 \%)(P=0.041)$.

Comparison of the clinical features between male and female subjects showed no significant difference in the 
Table 1 Overview of the TSC patient cohort

\begin{tabular}{|c|c|c|c|c|c|c|c|c|}
\hline & Total & $\begin{array}{l}\text { Mutation } \\
\text { identified }\end{array}$ & $\begin{array}{c}\text { Unclassified } \\
\text { variant }\end{array}$ & $\begin{array}{l}\text { No mutation } \\
\text { identified }\end{array}$ & $\begin{array}{c}\text { TSC1 } \\
\text { mutation }\end{array}$ & $\begin{array}{c}\text { TSC2 } \\
\text { mutation }\end{array}$ & De novo & Familial \\
\hline \multicolumn{9}{|c|}{ Patients with clinical information } \\
\hline Def. TSC & 276 & 235 & 14 & 27 & 53 & 182 & 92 & 50 \\
\hline Insuf. information & 15 & 4 & 1 & 10 & 1 & 3 & 1 & 1 \\
\hline Total & 291 & 239 & 15 & 37 & 54 & 185 & 93 & 51 \\
\hline No clinical information & 199 & 123 & 14 & 62 & 28 & 95 & 44 & 12 \\
\hline Total & 490 & 362 & 29 & 99 & 82 & 280 & 137 & 63 \\
\hline
\end{tabular}

Groups with a definite diagnosis of TSC (Def. TSC), insufficient clinical information for a definite diagnosis (Insuf. information) and without any clinical information (No clinical information) are shown. For each group, patients with mutations in the TSC1 and TSC2 genes in addition to patients with nucleotide changes that could not yet be classified as pathogenic (unclassified variant) are depicted. The number of patients with de novo mutations and of patients of familial cases (based on molecular analysis) are also shown.

Table 2 Comparison of the clinical features of TSC associated with gender

\begin{tabular}{|c|c|c|c|c|c|}
\hline & & & & & \\
\hline & $P / N$ & $\%$ & $P / N$ & $\%$ & P-values \\
\hline $\begin{array}{l}\text { Mental retardation } \\
\text { Seizures } \\
\text { Cortical tubers } \\
\text { SEN } \\
\text { SEGA }\end{array}$ & $\begin{array}{l}89 / 108 \\
110 / 121 \\
56 / 63 \\
84 / 93 \\
9 / 30\end{array}$ & $\begin{array}{l}83 \\
91 \\
89 \\
90 \\
30\end{array}$ & $\begin{array}{l}63 / 93 \\
90 / 104 \\
56 / 62 \\
73 / 82 \\
13 / 37\end{array}$ & $\begin{array}{l}68 \\
87 \\
90 \\
89 \\
35\end{array}$ & $\begin{array}{l}0.012^{*} \\
0.204 \\
0.512 \\
0.485 \\
0.429\end{array}$ \\
\hline $\begin{array}{l}\text { Renal AML } \\
\text { Renal cysts }\end{array}$ & $\begin{array}{l}31 / 74 \\
22 / 67\end{array}$ & $\begin{array}{l}42 \\
33\end{array}$ & $\begin{array}{l}33 / 70 \\
12 / 62\end{array}$ & $\begin{array}{l}47 \\
19\end{array}$ & $\begin{array}{l}0.321 \\
0.062\end{array}$ \\
\hline $\begin{array}{l}\text { Shagreen patch } \\
\text { Hypomelanotic macules } \\
\text { Forehead plaque } \\
\text { Facial angiofibromas } \\
\text { Ungual fibroma }\end{array}$ & $\begin{array}{l}30 / 56 \\
94 / 102 \\
23 / 53 \\
82 / 97 \\
28 / 64\end{array}$ & $\begin{array}{l}54 \\
92 \\
43 \\
85 \\
44\end{array}$ & $\begin{array}{l}30 / 61 \\
80 / 90 \\
17 / 50 \\
61 / 83 \\
17 / 59\end{array}$ & $\begin{array}{l}49 \\
89 \\
34 \\
74 \\
29\end{array}$ & $\begin{array}{l}0.386 \\
0.299 \\
0.219 \\
0.050^{\star} \\
0.063\end{array}$ \\
\hline $\begin{array}{l}\text { Retinal phakoma } \\
\text { Retinal depigmentation }\end{array}$ & $\begin{array}{l}26 / 69 \\
11 / 20\end{array}$ & $\begin{array}{l}38 \\
55\end{array}$ & $\begin{array}{l}11 / 55 \\
2 / 18\end{array}$ & $\begin{array}{l}20 \\
11\end{array}$ & $\begin{array}{l}0.025^{*} \\
0.005^{*}\end{array}$ \\
\hline $\begin{array}{l}\text { Gingival fibroma } \\
\text { Dental pits }\end{array}$ & $\begin{array}{l}10 / 17 \\
20 / 32\end{array}$ & $\begin{array}{l}59 \\
63\end{array}$ & $\begin{array}{l}5 / 23 \\
19 / 37\end{array}$ & $\begin{array}{l}22 \\
51\end{array}$ & $\begin{array}{l}0.019^{*} \\
0.246\end{array}$ \\
\hline Cardiac rhabdomyoma & $23 / 58$ & 40 & $20 / 53$ & 38 & 0.495 \\
\hline LAM & $0 / 19$ & 0 & $4 / 21$ & 19 & 0.146 \\
\hline
\end{tabular}

For each group, the first column shows the number of patients with the feature $(P)$ and the total number of patients examined $(N)$ and the second column shows the frequency of each clinical feature (\%). *Indicates statistically significant values. Median age was 14.0 years for males and 13.0 years for females $(P=0.467)$ (SEN, subependymal nodules; SEGA, subependymal giant cell astrocytoma; AML, angiomyolipoma; LAM, lymphangioleiomyomatosis).

median age of the two groups $(P=0.467)$ (Table 2). However, $75 \%$ of the clinical features analysed were more frequent in males. Mental retardation $(P=0.012)$, facial angiofibromas $(P=0.050)$, retinal phakomas $(P=0.025)$, retinal depigmentations $(P=0.005)$ and gingival fibromas $(P=0.019)$ were noted significantly more frequently in males. Lymphangioleiomyomatosis (LAM) was only detected in females (four cases). However, due to the low numbers of patients examined for LAM (12 males and 21 females), this difference was not significant $(P=0.146)$.

\section{Mutation analysis}

We identified pathogenic mutations in 362 patients (74\% of the total cohort), 82 mutations in the TSC1 gene and 280 in the TSC2 gene (23\% vs 77\%). The different types of TSC1 and TSC2 mutations are shown in Figure 1. In addition, we identified 20 different polymorphisms in the TSC1 gene and 56 in the TSC2 gene. In 29 cases (6\% of the total), we detected nucleotide changes in the TSC2 gene, but were unable to establish whether they were pathogenic. A complete 

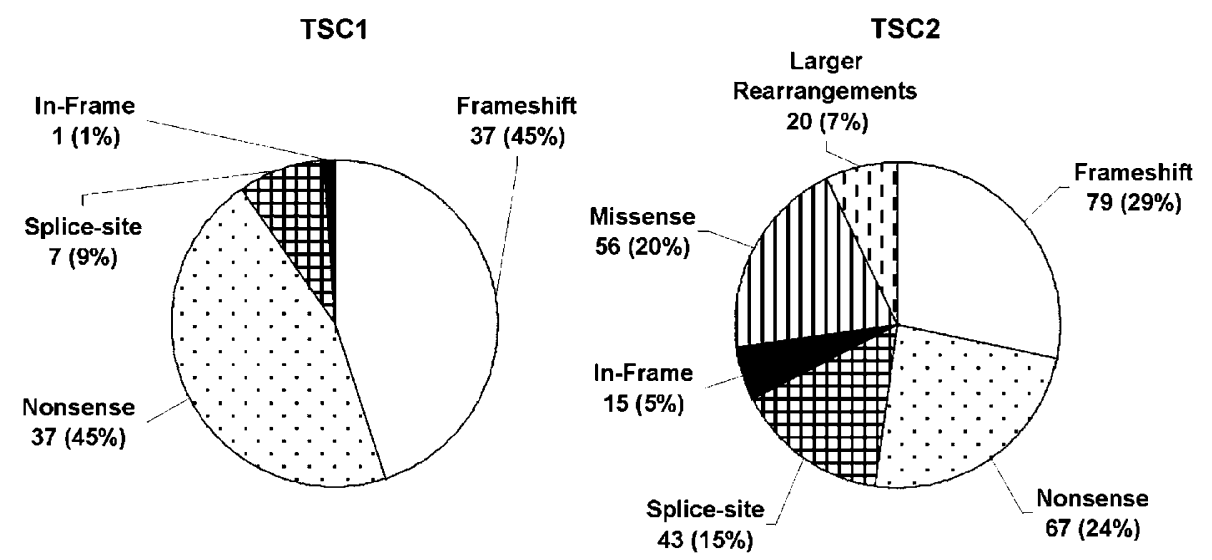

Figure 1 Types and frequencies of the mutations identified at the TSC1 and TSC2 loci. ( $\square$ frameshift mutations, $\mathbf{\square}$ in-frame deletions, 8 nonsense mutations, $\boxplus$ splice site mutations, $\square$ missense mutations, $\square$ large rearrangements).

overview of all the changes identified at both loci is available on request.

When possible, the parents of the patient were tested for the presence of a (putative) pathogenic mutation (Table 1). In 137 cases (22 TSC1 and 115 TSC2), the change identified in the patient was not present in the DNA of either parent. We defined these cases as de novo TSC. In 63 cases (20 TSC1 and 43 TSC2) the same pathogenic change was identified in the affected parent of the patient. In 10 cases, we could not rule out the possibility that a change identified in both the patient and another affected parent was nonpathogenic. In 181 cases the parents were not available for testing.

Four nucleotide changes in the TSC2 gene showed a possible effect on RNA splicing using three different splice-site prediction programs (www.fruitfly.org/seq tools/splice.html; www.cbs.dtu.dk/services/NetGene2 and www.genet.sickkids.on.ca/ ali/splicesitefinder.html). RTPCR analysis showed an incorrectly spliced TSC2 mRNA in three cases, confirming that the changes were pathogenic. In one case (IVS5 $+4 \mathrm{~A}>\mathrm{G}$ ) we were unable to detect any abnormally spliced variant. However, further investigation revealed that the expression of the mutant allele was severely reduced. In several cases the effects of TSC2 missense mutations on tuberin function were investigated. Missense changes that inactivated tuberin in vitro were classified as pathogenic. In cases where we could not demonstrate that missense and intronic changes were de novo or had an effect on splicing or the function of the tuberin-hamartin complex, we designated the changes as 'unclassified variants'.

Previously we described two cases of mosaicism at the TSC1 locus and four cases at the TSC2 locus in the parents of TSC patients. ${ }^{14}$ We have identified two additional cases of mosaicism in the TSC2 gene, one large de novo deletion in a patient ( $80 \%$ of the cells) and one nonsense mutation $(3112 \mathrm{C}>\mathrm{T}(\mathrm{R} 1032 \mathrm{X}))$ in the father of a familial case of TSC.
In 94 patients where both SSCP and DGGE were performed and a mutation was identified, 87 mutations (93\%) were identified by DGGE, and 79 mutations (84\%) were identified by SSCP. In 71 (76\%) cases the mutation was identified by both techniques. In the TSC2 gene, seven changes were detected by SSCP but not by DGGE, while seven changes in the TSC1 gene and eight changes in the TSC2 gene were detected by DGGE but not by SSCP. Large rearrangements of the TSC2 gene were identified by FISH and/or Southern blotting in 20 patients, accounting for 7\% of the TSC2 mutations. Of these, three were complete gene deletions, 12 were on one end of the gene, four were intragenic and one was an intragenic duplication. In $50 \%$ of cases the rearrangement was detected by both techniques.

Recurrent mutations were identified at both loci. The most common mutation was the 1850G $>$ A (R611Q) missense mutation in exon 16 of the TSC 2 gene which was detected in 10 unrelated patients and, together with the adjacent $1849 \mathrm{C}>\mathrm{T}$ (R611W) mutation that was detected in an additional four cases, accounted for 3.8\% of the mutations identified. The most frequently occurring TSC1 mutation, 1719C > T (R500X), was detected in six cases $(1.7 \%$ of the total mutations).

The distributions of small mutations within the TSC1 and TSC2 genes are shown in Figure 2. The most mutations in the TSC1 gene were identified in exon 15 (19/362; 5.2\% of total mutations) and in the TSC2 gene in exon 32 $(32 / 362 ; 8.8 \%$ of total mutations). No mutations were identified in exons 3, 19, 22 and 23 of the TSC1 gene or in exon $30 \mathrm{~B}$ of the TSC2 gene. We calculated the mean number of mutations (including splice site changes) per nucleotide for each exon of both genes. The overall mutation frequency was higher at the TSC2 locus $(0.045$ mutations per nucleotide) than at the TSC1 locus $(0.020$ mutations per nucleotide). However, as shown in Figure 2, there was considerable variation in the frequency of 

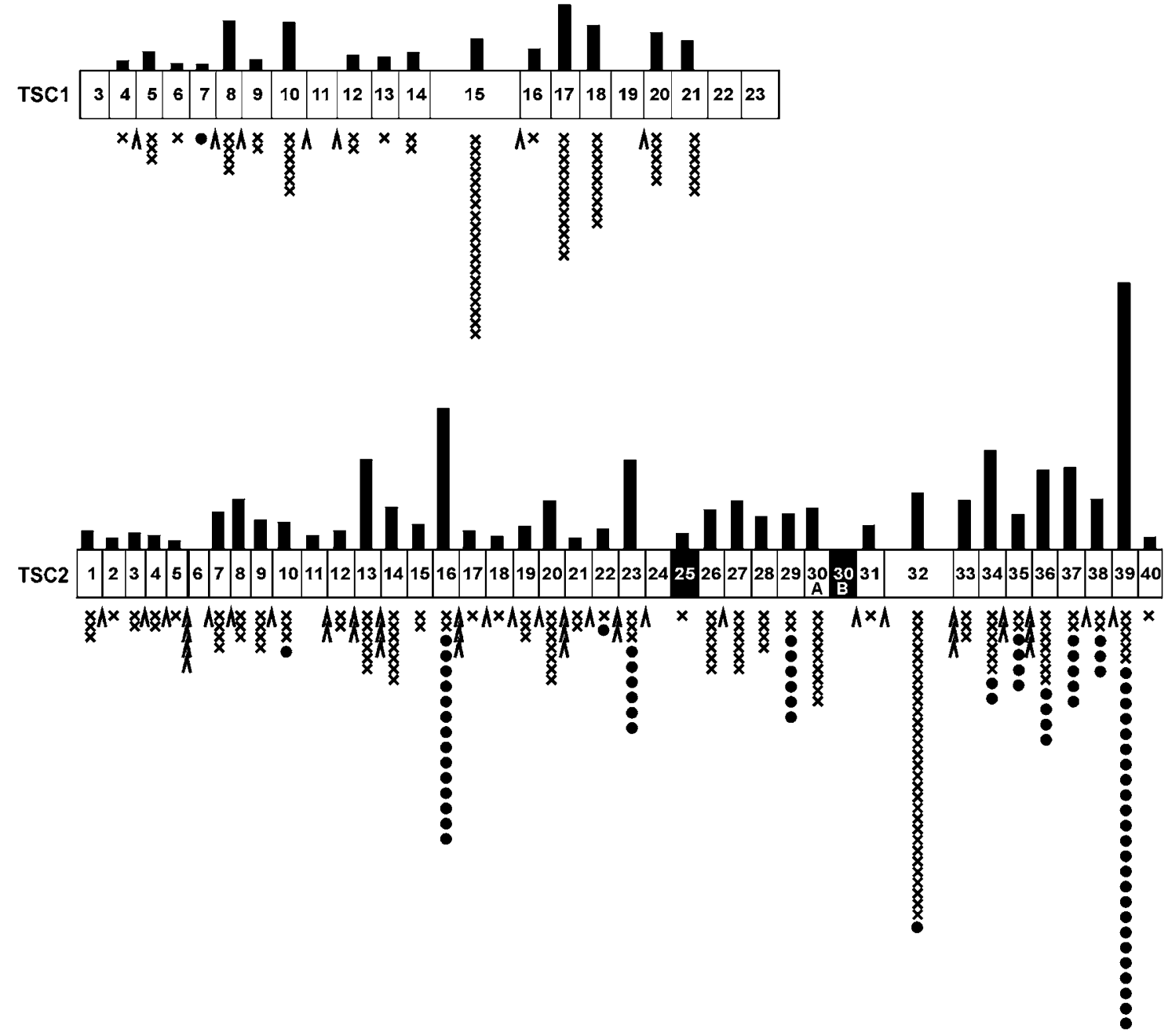

Figure 2 The distribution of small mutations along the TSC1 and the TSC2 genes ( $\bullet$ missense mutations and in-frame deletions, $\times$ nonsense and frameshift mutations, $\mathbf{V}$ splice site mutations). Alternatively spliced exons in the TSC2 gene are depicted in black. The mutation rate per nucleotide in each exon is graphed above each exon. The figures were calculated by dividing number of small mutations within the exon and the splicing mutations within five nucleotides in the $3^{\prime}$ and $5^{\prime}$ regions of the exon by the number of nucleotides of the exon plus 10 nucleotides for the surrounding intronic area. Note, exons are not drawn to scale.

mutations per exon. Exons 16 and 39 of the TSC2 gene had the highest frequency of mutations.

Exons 35-39 of the TSC2 gene encode the GTPase activating-protein (GAP) domain which is essential for tuberin function. We identified 64 small nucleotide mutations within these five exons, accounting for almost $18 \%$ of all the mutations identified. Interestingly, $46 \%$ of all the missense mutations and $93 \%$ of all the in-frame deletion mutations identified in the TSC2 gene were in this region.

\section{Phenotypic comparisons}

We obtained clinical information on 291 patients, of which 276 (95\%) fulfilled the definite TSC diagnostic criteria. In these 276 patients we identified 235 mutations; 53 in the
TSC1 gene and 182 in the TSC 2 gene, a mutation detection rate of $85 \%$. In 15 individuals where there was insufficient clinical evidence for a definite diagnosis of TSC, we identified four mutations. In two cases (mutations: TSC1 903C > T (R228X) and TSC2 4324delC), the patients were mentally retarded, had seizures, radiological brain abnormalities and hypomelanotic macules; in one case (mutation TSC2 $1135 \mathrm{C}>\mathrm{T}(\mathrm{Q} 373 \mathrm{X})$ ) dental pits and facial angiofibroma were noted in combination with epilepsy and mental retardation; and in one case (mutation TSC2 $4324 \mathrm{C}>\mathrm{T}(\mathrm{R} 1436 \mathrm{X}))$, subependymal nodules were the only clinical feature noted.

We compared patients with a TSC1 mutation to those with a TSC2 mutation (Tables $3 \mathrm{a}$ and $3 \mathrm{~b}$ ). The median age of the patients with a TSC1 mutation was not significantly 
Table 3a Comparison of the clinical features in TSC patients with and without mutations

\begin{tabular}{|c|c|c|c|c|c|c|c|c|c|c|c|c|c|c|c|c|c|c|c|c|}
\hline & \multicolumn{6}{|c|}{ TSC1 } & \multicolumn{6}{|c|}{ TSC2 } & \multirow{2}{*}{\multicolumn{2}{|c|}{$\begin{array}{c}\text { Mutations } \\
\text { Total }\end{array}$}} & \multirow{2}{*}{\multicolumn{2}{|c|}{ Familial }} & \multirow{2}{*}{\multicolumn{2}{|c|}{ De novo }} & \multirow{2}{*}{\multicolumn{2}{|c|}{$\begin{array}{l}\text { No Mutation } \\
\text { Identified }\end{array}$}} \\
\hline & \multicolumn{2}{|c|}{ Total } & \multicolumn{2}{|c|}{ Familial } & \multicolumn{2}{|c|}{ De novo } & \multicolumn{2}{|c|}{ Total } & \multicolumn{2}{|c|}{ Familial } & \multicolumn{2}{|c|}{ De novo } & & & & & & & & \\
\hline & $P / N$ & $\%$ & $P / N$ & $\%$ & $P / N$ & $\%$ & $P / N$ & $\%$ & $P / N$ & $\%$ & $P / N$ & $\%$ & $P / N$ & $\%$ & $P / N$ & $\%$ & $P / N$ & $\%$ & $P / N$ & $\%$ \\
\hline Mental retardation & $19 / 39$ & 49 & $8 / 12$ & 67 & $7 / 12$ & 58 & $129 / 156$ & 83 & $20 / 27$ & 74 & $53 / 63$ & 84 & $148 / 195$ & 76 & $28 / 39$ & 72 & $60 / 75$ & 80 & $8 / 22$ & 36 \\
\hline & & 91 & & 100 & & 87 & & 91 & & 82 & & 97 & & 91 & & & & & & 68 \\
\hline ers & & 83 & $6 / 6$ & 100 & $7 / 8$ & 88 & $93 / 104$ & 89 & $14 / 18$ & 78 & 37) & 95 & & 88 & $20 / 24$ & 83 & $44 / 47$ & 94 & $14 /$ & 78 \\
\hline & & 92 & $10 / 11$ & 91 & $11 / 12$ & 92 & & 91 & 16 & 73 & 58 & 100 & & 91 & & 79 & $69 / 70$ & 99 & & 82 \\
\hline SEGA & & 39 & $2 / 3$ & 67 & $1 / 4$ & 25 & $17 /$ & 29 & $2 / 6$ & 33 & $7 / 19$ & 37 & $22 / 7$ & 31 & $4 / 5$ & 44 & $8 / 23$ & 35 & $1 / 14$ & 7 \\
\hline enal AMU & 2 & 7 & $0 / 5$ & 0 & $0 / 1$ & 0 & $57 / 114$ & 50 & $10 / 23$ & 44 & $19 / 5$ & 37 & $59 / 14$ & 42 & $10 / 28$ & 36 & $19 / 62$ & 31 & $9 / 16$ & 56 \\
\hline & & 11 & & 20 & & 0 & & 28 & & 25 & & 27 & & 24 & & $\begin{array}{l}30 \\
24\end{array}$ & & 22 & & 31 \\
\hline & & 71 & $7 / 9$ & 78 & & 56 & & & & 27 & & & & & & & & & & 19 \\
\hline macules & $1 / 44$ & 93 & $12 / 12$ & 100 & $15 / 17$ & 88 & $134 / 146$ & 92 & $30 / 30$ & 100 & 60 & 91 & & 92 & $42 / 42$ & 100 & $75 /$ & 90 & & 74 \\
\hline & 6 & 27 & $2 / 8$ & 25 & $2 / 6$ & 33 & & 45 & $3 / 1$ & 23 & 15 & 48 & & 41 & $5 / 2$ & 24 & $17 \mid$ & 46 & & 13 \\
\hline & 2 & 71 & $8 / 12$ & 67 & $4 / 8$ & 50 & & 82 & $17 / 22$ & 77 & & 78 & & 80 & & 74 & & 75 & & 64 \\
\hline Ungual fibroma & $14 / 31$ & 45 & $2 / 8$ & 25 & $1 / 6$ & 17 & $30 / 89$ & 34 & $4 / 15$ & 27 & $15 / 39$ & 39 & $44 / 120$ & 37 & $6 / 23$ & 26 & $16 / 45$ & 36 & $4 / 15$ & 27 \\
\hline & & & & 0 & & 18 & & & $4 / 21$ & 19 & & & & & & & & & & 13 \\
\hline Retinal depigmentation & $0 / 8$ & 0 & $0 / 1$ & 0 & & 0 & $11 / 35$ & 31 & $1 / 4$ & 25 & $6 / 17$ & 35 & & 26 & $1 / 5$ & 20 & $6 / 19$ & 32 & $1 / 10$ & 10 \\
\hline Gingival & $5 / 1$ & 50 & $2 / 2$ & 100 & $1 / 2$ & 50 & $10 / 36$ & 28 & $0 / 1$ & 0 & $6 / 1$ & 32 & $15 / 46$ & 33 & $2 / 3$ & 67 & $7 / 21$ & 33 & $2 / 5$ & 40 \\
\hline & & 53 & & & & 40 & & 50 & & 57 & & (5) & & (1) & & 55 & & 50 & & 25 \\
\hline Cardiac rhabdomyoma & $10 / 24$ & 42 & $1 / 3$ & 33 & $6 / 11$ & 55 & $37 / 89$ & 42 & $7 / 17$ & 41 & $17 / 39$ & 44 & $47 / 113$ & 42 & $8 / 20$ & 40 & $23 / 50$ & 46 & $5 / 15$ & 33 \\
\hline
\end{tabular}

Familial represents the index patients of familial cases. For each group, the first column shows the number of patients with the feature $(P)$ and the total number of patients examined ( $N$ ) and the second column shows the frequency of each clinical feature (\%). The median age was 14.0 for TSC1 familial, 4.0 for TSC1 de nowo, 15.0 for TSC1 total 11.0 for TSC2 familial, 10.0 for TSC2 de novo, 12.0 for TSC2 total, 13.0 for mutations total, 9.5 for de novo, 11.5 for familial and 12.0 for no mutation identified groups (SEN, subependymal nodules; SEGA, subependymal giant cell astrocytoma; $\mathrm{AML}$, angiomyolipoma). 
Table $3 \mathbf{b}$ Significance $(P)$ values of the comparisons made between the different groups depicted in table $3 a$

\begin{tabular}{|c|c|c|c|c|c|c|c|c|}
\hline & $\begin{array}{l}\text { TSC1 } \\
\text { VS } \\
\text { TSC2 }\end{array}$ & $\begin{array}{c}\text { Familial } \\
\text { vs } \\
\text { de novo }\end{array}$ & $\begin{array}{c}\text { TSC2 familial } \\
\text { vs } \\
\text { TSC2 de novo }\end{array}$ & $\begin{array}{c}\text { TSC1 de novo } \\
\text { vs } \\
\text { TSC2 de novo }\end{array}$ & $\begin{array}{c}\text { TSC1 familial } \\
\text { Vs } \\
\text { TSC2 familial }\end{array}$ & $\begin{array}{c}\text { TSC1 familial } \\
\text { Vs } \\
\text { TSC1 de novo }\end{array}$ & $\begin{array}{c}\text { No mutation } \\
\text { vs } \\
\text { mutations total }\end{array}$ & $\begin{array}{c}\text { No mutation } \\
\text { vs } \\
\text { TSC1 total }\end{array}$ \\
\hline Mental retardation & $<0.001^{*}$ & 0.322 & 0.203 & 0.055 & 0.456 & 0.500 & $<0.001^{*}$ & 0.351 \\
\hline Seizures & 0.595 & 0.090 & $0.013^{*}$ & 0.139 & 0.125 & 0.780 & $0.001^{*}$ & $0.016^{*}$ \\
\hline Cortical tubers & 0.299 & 0.169 & 0.072 & 0.436 & 0.288 & 0.571 & 0.188 & 0.473 \\
\hline SEN & 0.550 & $0.001^{*}$ & $<0.001^{*}$ & 0.171 & 0.232 & 0.739 & 0.168 & 0.227 \\
\hline SEGA & 0.368 & 0.454 & 0.637 & 0.565 & 0.405 & 0.371 & 0.066 & 0.067 \\
\hline Renal AML & $<0.001^{*}$ & 0.634 & 0.399 & $0.011^{*}$ & 0.087 & $\star \star \star$ & 0.270 & $0.001^{*}$ \\
\hline Renal cysts & $0.050^{*}$ & 0.829 & 0.553 & $0.048^{*}$ & 0.657 & 0.313 & 0.515 & 0.100 \\
\hline Shagreen patch & $0.031^{*}$ & 0.637 & 0.085 & 0.623 & $0.035^{*}$ & 0.310 & $0.006^{*}$ & $0.001^{*}$ \\
\hline Hypomelanotic macules & 0.527 & $0.034^{*}$ & 0.098 & 0.519 & $* *$ & 0.335 & $0.006^{*}$ & $0.037^{*}$ \\
\hline Forehead plaque & 0.107 & 0.095 & 0.110 & 0.413 & 0.656 & 0.594 & $0.033^{*}$ & 0.277 \\
\hline Facial angiofibromas & 0.102 & 0.908 & 0.576 & 0.104 & 0.390 & 0.388 & 0.086 & 0.552 \\
\hline Ungual fibroma & 0.178 & 0.430 & 0.315 & 0.292 & 0.666 & 0.615 & 0.324 & 0.190 \\
\hline Retinal phakoma & $0.003^{*}$ & $0.030^{*}$ & 0.094 & 0.177 & 0.218 & 0.289 & 0.113 & 0.576 \\
\hline Retinal depigmentation & 0.073 & 0.538 & 0.593 & 0.456 & 0.800 & $\star *$ & 0.273 & 0.556 \\
\hline Gingival fibroma & 0.172 & 0.308 & 0.700 & 0.567 & 0.333 & 0.500 & 0.546 & 0.573 \\
\hline Dental pits & 0.528 & 0.795 & 0.571 & 0.500 & 0.652 & 0.643 & 0.317 & 0.329 \\
\hline Cardiac rhabdomyoma & 0.586 & 0.648 & 0.552 & 0.380 & 0.656 & 0.500 & 0.541 & 0.603 \\
\hline Median age & 0.321 & 0.226 & 0.921 & 0.364 & 0.119 & $0.027^{*}$ & 0.795 & 0.484 \\
\hline
\end{tabular}

angiomyolipoma). ${ }^{* *}$ Indicates where statistical analysis could not be performed, since the patients in both groups were either all positive or all negative for the analysed feature. 
different from the median age of those with a TSC2 mutation $(P=0.321)$. However, individuals with a TSC1 mutation were less often mentally retarded $(P<0.001)$, and renal angiomyolipomas $(P<0.001)$, renal cysts $(P=0.050)$, retinal phakomas $(P=0.003)$ and retinal depigmentations $(P=0.073)$ were all less frequent in the TSC1 mutation group. In contrast, shagreen patches occurred significantly more frequently in patients with a TSC1 mutation $(P=0.031)$. Interestingly, although the incidence of mental retardation was lower in the group of patients with a TSC1 mutation, the incidences of seizures $(P=0.595)$ and (sub)cortical tubers $(P=0.299)$ were not significantly different between the two groups.

We compared the phenotypes of individuals with a TSC2 (i) nonsense or frameshift mutation; (ii) missense mutation; (iii) large abnormality; and (iv) mutation in exons 35-39 (encoding the GAP domain). Both renal angiomyolipomas and renal cysts were more frequent in the group with nonsense and frameshift mutations (64\% and $37 \%$, respectively) than in the group with missense mutations (38\% and $24 \%, P=0.050$ and 0.367 ), or mutations in the GAP domain (39\% and $10 \%, P=0.032$ and 0.018$)$. Shagreen patches, forehead plaques, facial angiofibromas and ungual fibromas also occurred more often in the group of patients with a nonsense or frameshift mutation. The incidence of renal cysts was highest in the group of patients with large abnormalities of the TSC2 gene (out of 10 patients with clinical information, three patients with a whole gene deletion and one patient with an intragenic duplication had renal cysts). Mental retardation, seizures and subependymal nodules occurred most frequently in the group of patients with a mutation in the GAP domain.

Next, we compared the clinical features in the 50 familial cases to the clinical features in the 91 individuals with a de novo mutation (Tables $3 \mathrm{a}$ and $3 \mathrm{~b}$ ). The median age was higher in the familial cases $(P=0.226)$ and many clinical manifestations were less frequent. For subependymal nodules $(P=0.001)$ and retinal phakomas $(P=0.030)$ the differences were significant. In contrast, hypomelanotic macules were significantly more frequent in the familial cases $(P=0.034)$.

In our cohort, TSC2 mutations were associated with a more severe phenotypic spectrum than TSC1 mutations (see above and Tables $3 \mathrm{a}$ and $3 \mathrm{~b}$ ). In addition, the proportion of de novo cases with a TSC2 mutation (115/ $137 ; 84 \%)$ was higher than the proportion of familial cases with a TSC2 mutation $(43 / 63 ; 68 \%)(P=0.125)$, consistent with the more severe phenotypic spectrum observed in the de novo group. We compared the clinical features of familial and de novo cases with either a TSC1 or a TSC 2 mutation. As shown in Tables $3 \mathrm{a}$ and $3 \mathrm{~b}, 14$ out of 17 clinical features were more frequent in the group of patients with a de novo TSC2 mutation, than in the group with a familial TSC2 mutation. Seizures and subependymal nodules occurred significantly more frequently in the de novo group ( $P=0.013$ and $P<0.001$, respectively). The median age at referral was not significantly different between patients with a de novo TSC2 mutation and familial cases $(P=0.921)$. In contrast, patients with a familial TSC1 mutation were referred for DNA testing at a significantly older age than patients with a de novo TSC1 mutation $(P=0.027)$. Subependymal nodules, forehead plaques, retinal phakoma and cardiac rhabdomyoma were more frequent in the group with a de novo TSC1 mutation compared to the group with a familial TSC1 mutation.

Next, we compared the clinical features in the group of patients with a de novo TSC1 mutation to the group with a de novo TSC2 mutation. The majority of clinical features $(14 / 17)$ were seen at a lower frequency in the group with a de novo TSC1 mutation. Notably, mental retardation $(P=0.055)$, renal angiomyolipomas $(P=0.011)$ and renal cysts $(P=0.048)$ were less frequent in the group with a de novo TSC1 mutation. The median age at referral was not significantly different $(P=0.364)$.

The phenotypic differences between the familial cases with TSC1 and TSC2 mutations were less pronounced. The only significant difference was the higher frequency of shagreen patches in the group with TSC1 mutations $(P=0.035)$. The median age at referral was not significantly different between these two groups $(P=0.119)$.

The clinical manifestations in 27 individuals with clinically definite TSC, without an identified pathogenic mutation, were compared to patients with definite TSC and an identified pathogenic mutation (Tables $3 \mathrm{a}$ and $3 \mathrm{~b}$ ). Although the median age at referral in the two groups was not significantly different $(P=0.795)$, many of the clinical features including mental retardation $(P<0.001)$, seizures $(P=0.001)$, shagreen patches $(P=0.006)$, hypomelanotic macules $(P=0.006)$ and forehead plaques $(P=0.033)$ occurred at a significantly lower frequency in the group without an identified mutation. Other symptoms were also observed less frequently in this group, although the differences were not statistically significant $(p>0.05)$. Since the group of patients with a TSC1 mutation was also associated with a less severe phenotype, we compared the clinical features in the group without an identified mutation to the group with a TSC1 mutation. Most clinical features (13/17) were less frequent in the no mutation group. In particular, seizures $(P=0.016)$, shagreen patches $(P=0.001)$ and hypomelanotic macules $(P=0.037)$ occurred less frequently. In contrast, renal angiomyolipomas $(P=0.001)$ and renal cysts $(P=0.100)$ were more common in the no mutation group.

\section{Discussion}

Using a combination of DGGE, SSCP, FISH and Southern analysis, a total of 362 mutations were identified in 490 
patients (74\%); of these, 235 mutations were present in 276 patients fulfilling the TSC definitive diagnostic criteria (85\%), comparable to earlier studies. ${ }^{9,10,19}$ The detection rate dropped to $62 \%$ in the group of patients without any clinical information and to $27 \%$ in patients where the clinical evidence was not sufficient for a definitive diagnosis. This suggests that our cohort contains individuals that have been misdiagnosed with TSC and that, in many patients, good clinical work-up will be sufficient to make a definite diagnosis of TSC. However, in some cases, molecular genetic screening of patients with an incomplete clinical evaluation can assist in establishing the diagnosis.

We identified significant correlations between the presence of mental retardation and seizures and the presence of (sub)cortical tubers and mental retardation similar to previous studies. ${ }^{10}$ In addition, we identified significant correlations between the presence of seizures and (sub)cortical tubers, and between the presence of (sub)cortical tubers and subependymal nodules. Consistent with earlier work, we found a correlation between the incidence of renal angiomyolipomas and mental retardation in patients above 6 years of age. ${ }^{10,20}$

In our cohort, males with TSC were more likely to be mentally retarded and to have renal cysts, retinal lesions, facial angiofibromas, and gingival and ungual fibromas. Sex-linked differences in TSC-associated features have been described previously in humans and in animal models of the disease. For example, males with TSC have a greater risk of learning disorders and autism than females with the disease. ${ }^{21}$ In addition, sex hormones have been shown to affect the behaviour of cells derived from TSC-associated lesions $^{22}$ and in a mouse model of TSC, liver hemangiomas were more common and more extensive in female than in male mice. ${ }^{23}$ It will be interesting to investigate in more detail how sex hormones regulate the functions of tuberin and hamartin, and to determine how hormonal changes influence the TSC phenotype.

Previous studies of large numbers of TSC patients have used denaturing high-performance liquid chromatography (DHPLC) for the detection of small nucleotide changes in the TSC1 and TSC2 genes. $^{10,19}$ Although we used a different approach, we detected a similar spectrum of small TSC1 and TSC2 mutations. In 94 cases where both SSCP and DGGE were performed and a mutation was identified, DGGE had a slightly higher mutation detection rate (93\%) than SSCP (84\%). For various technical reasons, a small number of mutations at the TSC1 and TSC2 loci are likely to be missed by either SSCP or DGGE. Due to its higher capacity and sensitivity, DGGE is now the method of choice in our laboratory. Exons not amenable to DGGE analysis, are directly sequenced. To detect larger rearrangements at the TSC2 locus, we performed FISH and Southern blotting. Neither technique alone detected all of the rearrangements in our cohort and therefore we are currently developing a multiplex ligation-dependent probe amplification assay (MLPA).

We found a similar predominance of TSC2 mutations in both de novo and familial cases as previous studies. ${ }^{9-11,24}$ We also confirmed the lower frequency of de novo TSC1 mutations compared to de novo TSC2 mutations, which has been attributed to the smaller size and less complex structure of the TSC1 genomic locus, and the rarity of large DNA rearrangements, missense and splice site mutations at this locus. ${ }^{24}$ It has also been suggested that TSC1 mutations are associated with a less severe TSC phenotype. ${ }^{9,10}$ In our cohort, individuals with a TSC1 mutation were less likely to be mentally retarded than patients with a TSC2 mutation, even though the incidence of tubers and seizures in the group of patients with a TSC1 mutation was not significantly lower. Previous studies have suggested that there is a correlation between tuber number and mental retardation in TSC patients and that, on average, fewer tubers and subependymal nodules are found in patients with a TSC1 mutation. ${ }^{10}$ We were unable to obtain data on the number of tubers to determine whether this could explain the lower incidence of mental retardation in the patients with a TSC1 mutation in our cohort. Renal and retinal abnormalities occurred less frequently in the group of patients with a TSC1 mutation, consistent with other studies. ${ }^{9,10}$ Although the incidence of dermatological abnormalities was not significantly reduced in this group, shagreen patches were present significantly more often. The lower incidence of renal abnormalities in this group, in contrast to the approximately equal incidence of brain and skin lesions, is consistent with the proposal that at least some of the phenotypic differences between these two groups is due to a differential role of tuberin and hamartin in renal cell growth control. $^{25}$

We identified a lower proportion of TSC2 mutations in familial cases of TSC than in de novo cases (68vs 84\%). We compared the clinical features of both familial and de novo cases of TSC caused by mutations to either gene. Similar to the comparison of the cohort as a whole (see above), de novo TSC1 mutations were associated with a lower frequency of clinical features than de novo TSC2 mutations. In familial cases, however, there was less difference between the clinical features of the groups of patients with a TSC1 or TSC2 mutation. This suggested that familial cases with a TSC2 mutation are associated with a milder TSC phenotype than de novo TSC2 cases, which was supported by a comparison of the frequencies of their clinical features. We did not detect any significant phenotypic differences between familial and de novo cases with a TSC1 mutation.

The mutation frequency, as calculated per exonic nucleotide, at the TSC2 locus was higher compared to that at the TSC1 locus and, not surprisingly, the exons with the highest frequencies of mutation (TSC2 exons 16 and 39) 
both contained recurrent mutations. The high proportion of nontruncating mutations in exons 35-39 of the TSC2 gene supports previous findings that the GAP domain of the TSC2 gene is a target for missense mutations. ${ }^{26}$

The frequencies of neurological symptoms and some brain lesions were slightly higher and the incidence of renal abnormalities was lower in patients with mutations in exons 35-39 than in other groups. These differences were not significant. Overall, we could not confirm that mutations to the tuberin GAP domain are associated with a more severe phenotype. ${ }^{26}$ The high incidence of renal cysts in the group with large abnormalities is consistent with the disruption of the adjacent PKD1 gene in several of these cases. ${ }^{1}$

In 27 patients with definite TSC, we did not identify a mutation in either the TSC1 or TSC2 gene. Most clinical features, with the exception of renal abnormalities and gingival fibromas, occurred less frequently in this group of patients compared to the group with a mutation. It is possible that mutations in regulatory regions that impair gene transcription or translation account for the milder phenotypes of these cases. Somatic mosaicism may also explain why in some patients no mutations were detected. ${ }^{10}$ Recently, Roberts et al ${ }^{27}$ reported somatic mosaicism in $3 \%$ of patients diagnosed with definite TSC. We have identified eight cases of somatic mosaicism: only one patient and seven parents of a patient. Since we stopped using an allele specific method for family studies, it is possible that some mosaic parents have been missed. It is likely that there are more mosaic patients in our cohort with a degree of mosaicism below the detection limit of the current screening protocol.

In summary, in our diagnostic setting of TSC mutation analysis an overall mutation detection rate of $74 \%$ was obtained; a pathogenic mutation was identified in $85 \%$ of definite TSC patients. Our study reveals that several clinical manifestations of TSC occur more frequently in males. In addition, TSC2 mutations are associated with a more severe phenotype than TSC1 mutations, and the group of patients with a de novo TSC2 mutation have a more severe phenotypic spectrum than the group of patients with a familial TSC2 mutation. The identification of a TSC1 or a TSC2 mutation will not only confirm the diagnosis of TSC but will also be important for both clinical management and possible therapeutic or preventive measures.

\section{Acknowledgements}

We thank all the patients who contributed DNA samples for analysis, and the referring clinicians. Gregory Asmawidjaja, Anita Jankie, Kim van Zijl, Rodney Blenham and Margje van Schaik are thanked for technical assistance. This work was supported by the Nationaal Epilepsie Fonds, Noortman BV, the Nederlandse Organisatie voor Wetenschappelijk Onderzoek and the Department of Clinical Genetics at the Erasmus MC.

\section{References}

1 Gomez M, Sampson J, Whittemore V: The Tuberous Sclerosis Complex. Oxford, England, Oxford University Press, 1999.

2 Henske EP, Scheithauer BW, Short MP et al: Allelic loss is frequent in tuberous sclerosis kidney lesions but rare in brain lesions. Am J Hum Genet 1996; 59: 400-406.

3 Nellist M, van Slegtenhorst MA, Goedbloed M, van den Ouweland AM, Halley DJ, van der Sluijs P: Characterization of the cytosolic tuberin-hamartin complex. Tuberin is a cytosolic chaperone for hamartin. J Biol Chem 1999; 274: 35647-35652.

4 Garami A, Zwartkruis FJ, Nobukuni T et al: Insulin activation of Rheb, a mediator of mTOR/S6K/4E-BP signaling, is inhibited by TSC1 and 2. Mol Cell 2003; 11: 1457-1466.

5 Identification and characterization of the tuberous sclerosis gene on chromosome 16: The European Chromosome 16 Tuberous Sclerosis Consortium. Cell 1993; 75: 1305-1315.

6 van Slegtenhorst M, de Hoogt R, Hermans C et al: Identification of the tuberous sclerosis gene TSC1 on chromosome 9q34. Science 1997; 277: 805-808.

7 van Slegtenhorst M, Verhoef S, Tempelaars A et al: Mutational spectrum of the TSC1 gene in a cohort of 225 tuberous sclerosis complex patients: no evidence for genotype-phenotype correlation. J Med Genet 1999; 36: 285-289.

8 Au KS, Rodriguez JA, Finch JL et al: Germ-line mutational analysis of the TSC2 gene in 90 tuberous-sclerosis patients. Am J Hum Genet 1998; 62: 286-294.

9 Jones AC, Shyamsundar MM, Thomas MW et al: Comprehensive mutation analysis of TSC 1 and TSC 2 and phenotypic correlations in 150 families with tuberous sclerosis. Am J Hum Genet 1999; 64: $1305-1315$.

10 Dabora SL, Jozwiak S, Franz DN et al: Mutational analysis in a cohort of 224 tuberous sclerosis patients indicates increased severity of TSC2, compared with TSC1, disease in multiple organs. Am J Hum Genet 2001; 68: 64-80.

11 Niida Y, Lawrence-Smith N, Banwell A et al: Analysis of both TSC1 and TSC2 for germline mutations in 126 unrelated patients with tuberous sclerosis. Hum Mutat 1999; 14: 412-422.

12 Langkau N, Martin N, Brandt R et al: TSC1 and TSC2 mutations in tuberous sclerosis, the associated phenotypes and a model to explain observed TSC1/ TSC2 frequency ratios. Eur J Pediatr 2002; 161: $393-402$.

13 Cheadle JP, Reeve MP, Sampson JR, Kwiatkowski DJ: Molecular genetic advances in tuberous sclerosis. Hum Genet 2000; 107: $97-114$.

14 Verhoef S, Bakker L, Tempelaars AM et al: High rate of mosaicism in tuberous sclerosis complex. Am J Hum Genet 1999; 64: $1632-1637$.

15 Eussen BH, Bartalini G, Bakker L et al: An unbalanced submicroscopic translocation $\mathrm{t}(8 ; 16)(\mathrm{q} 24.3 ; \mathrm{p} 13.3)$ pat associated with tuberous sclerosis complex, adult polycystic kidney disease, and hypomelanosis of Ito. J Med Genet 2000; 37: 287-291.

16 Nellist M, Verhaaf B, Goedbloed MA, Reuser AJ, van den Ouweland AM, Halley DJ: TSC2 missense mutations inhibit tuberin phosphorylation and prevent formation of the tuberinhamartin complex. Hum Mol Genet 2001; 10: 2889-2898.

17 Nellist M, Sancak O, Goedbloed MA et al: Distinct effects of single amino-acid changes to tuberin on the function of the tuberinhamartin complex. Eur J Hum Genet 2005; 13: 59-68.

18 Roach ES, DiMario FJ, Kandt RS, Northrup H: Tuberous Sclerosis Consensus Conference: recommendations for diagnostic evaluation. National Tuberous Sclerosis Association. J Child Neurol 1999; 14: $401-407$.

19 Jones AC, Sampson JR, Hoogendoorn B, Cohen D, Cheadle JP: Application and evaluation of denaturing HPLC for molecular genetic analysis in tuberous sclerosis. Hum Genet 2000; 106: $663-668$.

20 O'Callaghan FJ, Noakes M, Osborne JP: Renal angiomyolipomata and learning difficulty in tuberous sclerosis complex. J Med Genet 2000; 37: 156-157. 
21 Smalley SL, Tanguay PE, Smith M, Gutierrez G: Autism and tuberous sclerosis. I Autism Dev Disord 1992; 22: 339-355.

22 Yu J, Astrinidis A, Howard S, Henske EP: Estradiol and tamoxifen stimulate lymphangiomyomatosis-associated angiomyolipoma cell growth and activate both genomic and non-genomic signaling pathways. Am J Physiol Lung Cell Mol Physiol 2003.

23 Kwiatkowski DJ, Zhang H, Bandura JL et al: A mouse model of TSC1 reveals sex-dependent lethality from liver hemangiomas, and up-regulation of p70S6 kinase activity in Tsc1 null cells. Hum Mol Genet 2002; 11: 525-534.
24 Kwiatkowski DJ: Tuberous sclerosis: from tubers to mTOR. Ann Hum Genet 2003; 67: 87-96.

25 Yeung RS: Multiple roles of the tuberous sclerosis complex genes. Genes Chromosomes Cancer 2003; 38: 368-375.

26 Maheshwar MM, Cheadle JP, Jones AC et al: The GAP-related domain of tuberin, the product of the TSC2 gene, is a target for missense mutations in tuberous sclerosis. Hum Mol Genet 1997; 6: $1991-1996$

27 Roberts PS, Dabora S, Thiele EA, Franz DN, Jozwiak S, Kwiatkowski DJ: Somatic mosaicism is rare in unaffected parents of patients with sporadic tuberous sclerosis. J Med Genet 2004; 41: e69. 